\title{
Laser-based satellite positioning system for space gravitational wave detector DECIGO
}

Mitsuru Musha, Mika Tajiri, Kei Akami, Aru Suemasa, Yuichi Takeuchi

Mitsuru Musha, Mika Tajiri, Kei Akami, Aru Suemasa, Yu-ichi Takeuchi, "Laser-based satellite positioning system for space gravitational wave detector DECIGO," Proc. SPIE 11852, International Conference on Space Optics - ICSO 2020, 1185240 (11 June 2021); doi: 10.1117/12.2599634

SPIE Event: International Conference on Space Optics - ICSO 2021, 2021, Online Only 


\section{International Conference on Space Optics-ICSO 2020}

Virtual Conference

30 March-2 April 2021

Edited by Bruno Cugny, Zoran Sodnik, and Nikos Karafolas
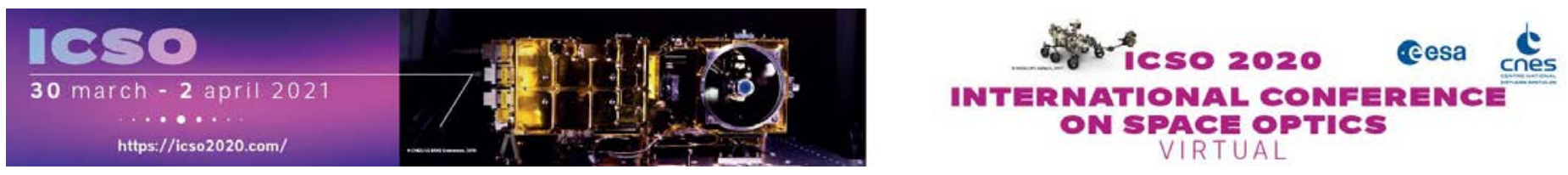

\section{Laser-based satellite positioning system for space gravitational wave detector DECIGO}

\section{Cesa issopreatings denes}




\title{
Laser-based satellite positioning system for space gravitational wave detector DECIGO
}

\author{
Mitsuru Musha*, Mika Tajiri, Kei Akami, Aru Suemasa, Yu-ichi Takeuchi \\ Institute for Laser Science, University of Electro-communications, 1-5-1 Chofugaoka, Chofu-shi, \\ Tokyo 1828585, Japan
}

\begin{abstract}
We propose the inter-satellite laser positioning system for precision formation flying. Our system consists of a single frequency laser and acousto-optic deflectors, and the remote spacecraft can obtain their angle information by frequency counting of the laser beat signal. The accuracy of the angle measurement is evaluated to be $0.004 \mathrm{mrad}$, and we expand this system to two-dimensional angle measurement. The distance between $\mathrm{S} / \mathrm{Cs}$ can be also measured by using microwave phase in the detected beam, and length resolution of $6.25 \mathrm{~m}$ is obtained from our preliminary test. We plan to apply this system to the initial alignment of three $\mathrm{S} / \mathrm{Cs}$ for the precision formation flying of Japanese space gravitational wave detector DECIGO project.
\end{abstract}

Keywords: laser ranging, formation flying, angle measurement, gravitational wave detector

\section{INTRODUCTION}

Precision formation flying of the space crafts (S/C) is one of the essential techniques for the future space science missions such as very long-baseline infrared interferometers [1][2], a satellite gravimetry mission [3], long-focal-length X-ray or gamma ray telescopes [4][5][6] and much larger-size satellite network for testing general relativity[7][8]. In these mission, effective aperture size or focal length of the telescope is dramatically expanded to improve angle or image resolution. Among these formation flying missions, space gravitational wave detectors would have highest degree of technical difficulties from their much longer formation size and precision.

Gravitational wave had been predicted in 1916 by A.Einstein in his general theory of relativity which would be generated by the acceleration of mass such as explosion of super novae or inspiral and coalescence of blackhole binaries. Due to its extremely small strain of lower than $\delta l / l<10^{-23}$, direct detection of gravitational wave is very difficult. In 2016, the first direct detection of gravitational wave had been achieved by advanced LIGO [9], and successive detections have been reported from ground-based gravitational wave detectors. The ground-based gravitational wave detector is a Fabry-Perot Michelson laser interferometer with the arm length of 3-4 km, and has strain sensitivity of $10^{-23}$ level around $10 \mathrm{ko} 1 \mathrm{kHz}$. Since the detection band of the ground-based gravitational wave detector at lower frequency range is limited by its finite arm length and seismic noise from the ground, space gravitational wave detectors are planned to detect lower frequency gravitational wave which have much longer armlength and are operating in seismic noise free condition. Europe and US space gravitational detector named LISA consists of tree S/C each of which separate more than 2,500,000 km and are triangle-shaped constellation flying [10]. In LISA the distortion of space is measured by using laser transponder for detecting gravitational wave at lower frequency around $1 \mathrm{mHz}$. In Japan, we promote another space gravitational mission named DECIGO (DECi-hertz Interferometer Gravitational wave Observatory) consists of three S/Cs forming equilateral triangle-shaped laser interferometer with the arm length of $1000 \mathrm{~km}$, whose conceptual design is shown in Fig.1 [11]. DECIGO contains three differential 1000-km Fabry-Perot laser interferometer with the arm length of $1000 \mathrm{~km}$ and the including angle of 60 degree in its triangle-shape formation, and strain sensitivity of $\mathrm{dL} / \mathrm{L}<10^{-23}$ at the frequency range between 0.1 to $1 \mathrm{~Hz}$ for detecting gravitational wave from the inspiral of intermediate-mass blackhole binaries or primordial background gravitational wave from the early universe.

*musha@ils.uec.ac.jp; phone 8142 443-5705; fax 8142 485-8960 
DECIGO is planned to launch in mid 2030s. Before launching DECIGO, we plan another milestone mission named BDECIGO, which is down-sized mission of DECIGO with the arm length of $100 \mathrm{~km} \mathrm{[12].}$

In order to obtain such high strain sensitivities, the length of each interferometer arm should be identical with the length difference of lower than $0.1 \%$ for improving common-mode noise reduction (CMNR) because higher CMNR reduces the influence of the intensity and frequency noise of the light source on the strain sensitivity of the interferometer. For initial alignment of three S/Cs after releasing them from the launching vehicle and keeping their relative position in the observation, we proposed novel satellite positioning system based on the combination of a single-frequency laser, acoustooptic modulator and frequency counting. In the previous conference (ICSO2018), the basic idea of our angle measurement system was introduced [13]. Our angle measurement system has been expanded to add two-dimensional angle measurement and length measurement functions, the details of which are presented in the current document.

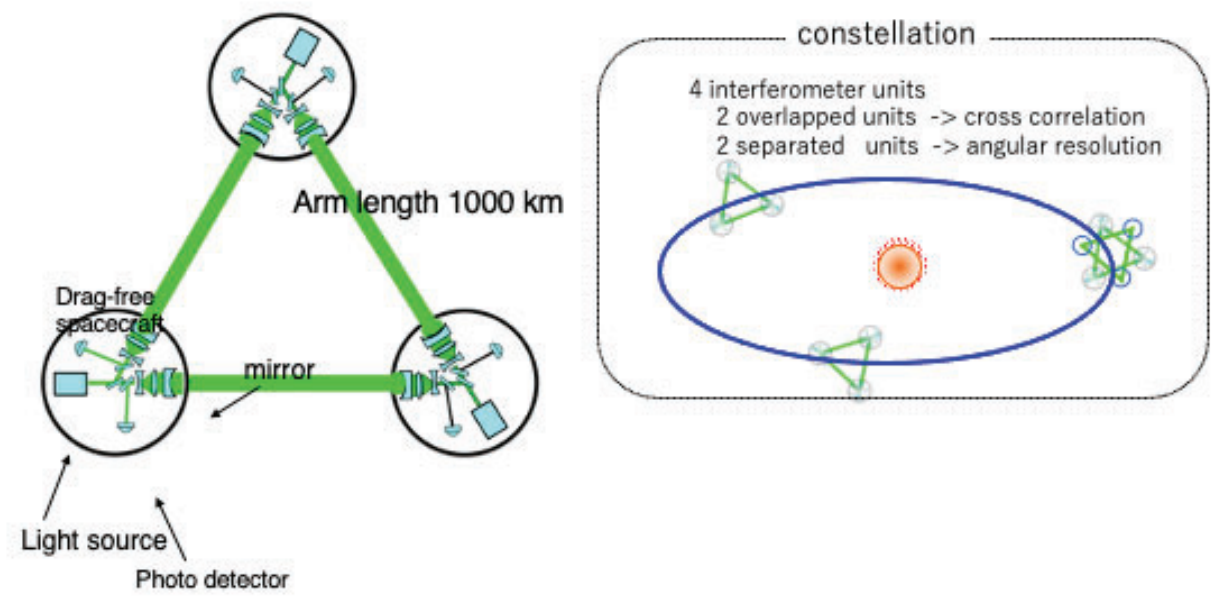

Figure 1 Conceptual design of DECIGO. In the final stage four units of DECIGO will launched around-synchronous orbits for improving angle resolution and sensitivity

\section{ANGLE MEASUREMENT}

\section{1 principle}

Acousto-optic deflector (AOD) is the optical diffraction device which utilizes the interference between acoustic wave and light. When microwave signal is applied to AOD crystal, piezo-electric transducer attached to AOD crystal generates compression wave inside crystal to make Bragg grating. The laser beam introduced into AOD generates $1^{\text {st }}$ diffraction beam whose diffraction angle, $\theta$, is proportional to the driving frequency of AOM, $f_{\mathrm{m}}$ as

$\theta_{\mathrm{m}}=\left(\lambda / \mathrm{V}_{\mathrm{a}}\right) f_{\mathrm{m}}$

$V_{\mathrm{a}}$ and $\lambda$ in this equation are the acoustic velocity in the crystal and the wavelength of the diffracted laser, respectively. Since the frequency of the $1^{\text {st }}$ diffracted beam is shifted as $f_{\mathrm{m}}$ due to Doppler effect, the frequency shift of the diffracted beam is proportional to the diffraction angle.

The leader S/C emits the first diffracted beam along the reference line on which the follower S/C should be located, and the diffraction angle is scanned by changing driving frequency, $f_{\mathrm{m}}$. When the follower $\mathrm{S} / \mathrm{C}$ detects scanned diffracted beam, it obtains the angle information from the frequency of detected diffraction beam. The frequency shift is obtained as the beat note frequency in the diffracted beam 


\subsection{Experimental set-up}

Schematic diagram of the angle measurement system is shown in Fig.2. Laser light with the wavelength of $515 \mathrm{~nm}$ is introduced into a polarization beam splitter (PBS1) to divide into signal and local beam. The power ratio of the signal and the local beam is controlled by a half-wave plate inserted before PBS1. The signal beam is introduced into AOD whose $1^{\text {st }}$ diffracted bean is selected by a spatial filter and is propagated toward the remote site. The local beam from PBS1 goes into a local arm 1 where acousto-optic modulator (AOM1) is located. AOD1 and AOM1 are driven by the same frequency, $f_{\mathrm{m}}$, generated by a computer-controlled direct digital synthesizer (DDS). The $1^{\text {st }}$ diffracted local beam from AOM1 is reflected by a concaved mirror back into AOM1 again to generate $1^{\text {st }}$ diffraction beam. By optimizing beam mode and alignment, this double-pass configuration makes $2 f_{\mathrm{m}}$-frequency-shifted local beam which goes back to the PBS1 along the same line as of the input local beam. A quarter-wave plate (QWP1) rotates the polarization of the local beam from P to S so that the returned local beam is reflect by PBS1 into the local arm2. In the local arm 2, the local beam is reflected back into PBS1 to collinearly overlap with the signal beam by using a combination of QWP2 and mirror, and is introduced into AOD1 to make diffraction beam with the signal beam. Therefore, the remote site detects both diffracted signal beam with frequency of $f_{\mathrm{s}}=f_{0}+f_{\mathrm{m}}$ and the local beam with the frequency of $f_{\mathrm{L}}=f_{0}+3 f_{\mathrm{m}}$ to obtain $2 f_{\mathrm{m}}$ beat signal between them.

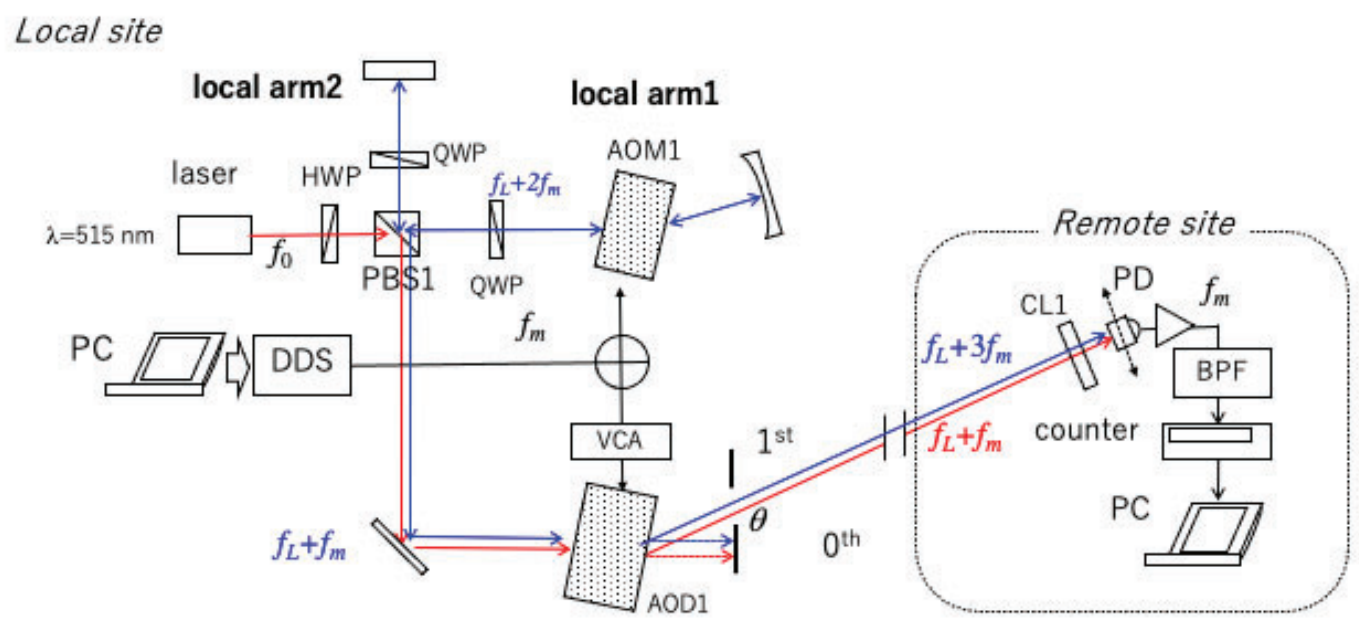

Figure 2 Experimental set-up of the angle measurement system. Red and blue arrows indicate signal and local beam, reflectively. At the remote end, photo detector detects beat note between local and signal beam. DDS: direct signal synthesizer, VCA: voltage-controlled oscillator, BPF: band-pass filter.

At the remote site, the diffracted beam is detected by a fast photo detector for counting beat note frequency and also dc detected power. As the diffraction efficiency of the AOD varies with the driving frequency, the power stabilization servo is applied to AOD1. A part of the diffracted light is pick off, and the power fluctuations of the diffracted beam is suppressed by controlling applied microwave power to AOD. In activating negative feedback servo loop, the diffracted beam power is kept constant while the beam angle is scanned.

\subsection{Two-dimensional scanning}

For searching the follower satellite, two-dimensional beam scanning is necessary. Therefore, another acousto-optic deflector (AOD2) is added. AOD2 is set just after AOD1 whose crystal axis is orthogonal to that of AOD1, and is driven by $f_{\mathrm{n}}$ with another DDS. The beam propagation can be controlled to arbitrary direction on the 2-D plane by setting $f_{\mathrm{m}}$ and $f_{\mathrm{n}}$ independently. However, the remote $\mathrm{S} / \mathrm{C}$ should get two information for determine his position on the 2-D plane (angle information of $\mathrm{x}$ and $\mathrm{y}$ axis). In order to carry two frequency information, $f_{\mathrm{m}}$ and $f_{\mathrm{n}}$, on one diffracted beam, another acousto-optic modulator AOM2 is added in the local arm 2 which is driven with the frequency of $f_{\mathrm{n}}$. The local beam from 
the local arm 1 is introduced into AOM2, and both $0^{\text {th }}$ and $1^{\text {st }}$ diffracted beam is selected by a spetial filter, and they reflect back into the AOM2 again and only one beam is selected by a spatial filter which go back collinearly with the input beam $\left(0^{\text {th }}\right.$-to $0^{\text {th }}$ and $1^{\text {st }}$ to $1^{\text {st }}$ diffracted beam $)$. In the consequence, there are three frequency components staying in the diffracted beam from AOD $1, f_{0}+f_{\mathrm{m}}+f_{\mathrm{n}}, f_{0}+3 f_{\mathrm{m}}+f_{\mathrm{n}}$ and $f_{0}+3 f_{\mathrm{m}}+3 f_{\mathrm{n}}$. At the remote site, three beat notes with the frequency of $2 f_{\mathrm{m}}, 2 f_{\mathrm{n}}$ and $f_{\mathrm{m}}+2 f_{\mathrm{n}}$ are obtained. Therefore, the remote site can know their 2-D angle information from $2 f_{\mathrm{m}}, 2 f_{\mathrm{n}}$ beat frequencies by detecting one scanned beam.

\section{3 result and discussion}

For the preliminary test, we set a three-fold 10-m optical path between the local and remote site, and the one-dimensional angle resolution of this system is evaluated. At the local site, the driving frequency of AOD $1, f_{\mathrm{m}}$, is swept for $5 \mathrm{MHz}$ around $75 \mathrm{MHz}$ to scan diffraction beam angle. The diffracted beam is detected at the remote site, $10.35 \mathrm{~m}$ apert from the local site, and the detection system moves for $2 \mathrm{~mm}$ along the scanning line on the vertical plane to the optical axis. The detection system has an aperture with the diameter of $1 / 2$ " and the transmitted light through the aperture is focused on a photo detector (Hamamatsu S5978) with a 50-mm focal length lens. The detection system obtains both the beat note frequency and DC detected power continuously during beam scanning, and the center frequency of the beat signal is determined at the peak DC voltage. The frequency data are acquired in every $0.5 \mathrm{~mm}$ steps. From the 5 times averaging data, the angle precision of $0.0035 \mathrm{mrad}$ with the angle resolution of $0.017 \mathrm{mrad}$ are obtained. The angle resolution limit in this measurement comes from the pointing fluctuation of the diffracted beam which is caused from the vibration of the mirrors and air turbulences. The angle resolution would be improved by using direct path under the vacuum conditions. Theoretical angle resolution limit is determined by the resolution of the frequency count, which comes from the SNR of the beat signal. In order to obtain the angle resolution of $0.001 \mathrm{mrad}$, the frequency resolution of $10 \mathrm{kHz}$ is required. For satisfying this resolution limit, more than $5 \mu \mathrm{W}$ of the detected power is necessary. In the case of DECIGO, beam diameter from the leader satellite is expanded to $28.5 \mathrm{~m}$ at the follower satellite due to diffraction, and $10 \mathrm{~mW}$ of the beam power is calculated to be high enough for obtaining angle resolution of $0.001 \mathrm{mrad}$.

\section{LENGTH MEASUREMENT}

For acquiring or keeping precision formation flying in DECIGO project, not only angle but also distance between spacecrafts should be measured. Currently, time-of-flight method (TOF) is widely used for the length measurement in space [14], and recent progress of optical frequency comb improves the resolution of long-distance measurement [15] . In these methods, ultra-short pulse light source and precision timing or phase measurement are required. We propose length measurement method which can be installed in our angle measurement system. Let me introduce the principle of our length measurement method. When the electric-magnetic (EM) wave with the wavelength of $\lambda$ is travelling from the local to the remote site, the distance between two sites, $L$, can be written as

$$
L=\left(N+\frac{\phi}{2 \pi}\right) \lambda=\left(N+\frac{\phi}{2 \pi}\right) \frac{c}{f}
$$

where $c, f, N$ and $\phi$ are speed of light, frequency of EM wave, number of waves in $L$ and residual EM phase, respectively. When laser frequency is changed from $f_{1}$ to $f_{2}$, this equation is written as

$$
\begin{aligned}
& L=\left(N_{1}+\frac{\phi_{1}}{2 \pi}\right) \frac{c}{f_{1}}=\left(N_{2}+\frac{\phi_{2}}{2 \pi}\right) \frac{c}{f_{2}} \\
& f_{1}-f_{2}=\left\{\left(N_{1}-N_{2}\right)+\frac{\left(\phi_{1}-\phi_{2}\right)}{2 \pi}\right\} \frac{c}{L}
\end{aligned}
$$


Therefore, the length $\mathrm{L}$ can be obtained from the number difference $\Delta N$ and phase difference $\Delta \phi$ as

$$
L=\frac{\left(N_{1}-N_{2}\right)+\frac{\left(\phi_{1}-\phi_{2}\right)}{2 \pi}}{f_{1}-f} c=\frac{\Delta N+\Delta \phi / 2 \pi}{\Delta f} c
$$

When the frequency varies continuously for $\Delta f, \Delta N$ and $\Delta \phi$ can be measured from the variation of the phase change at the remote end.

According to this principle, the distance between two sites can be measured as follows

The EM wave is travelling toward the remote site, and at the remote site, the detected EM wave is mixed with local oscillator to demodulate for obtaining the phase at the remote end. As the frequency of EM wave is swept from $f_{1}$ to $f_{2}$, the phase change at the remote end can be obtained by demodulating the transmitted EM wave synchronously. From the cyclic change of the phase, we can obtain $\Delta N$.

The proof-of-principle test has been done whose schematic is shown in Fig.3(a).

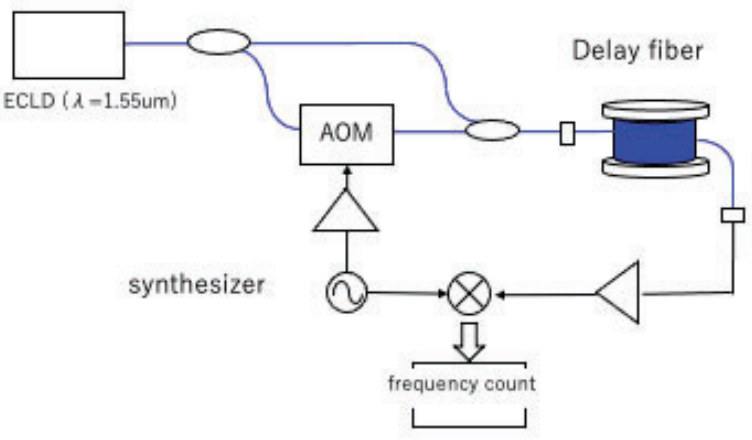

(a)

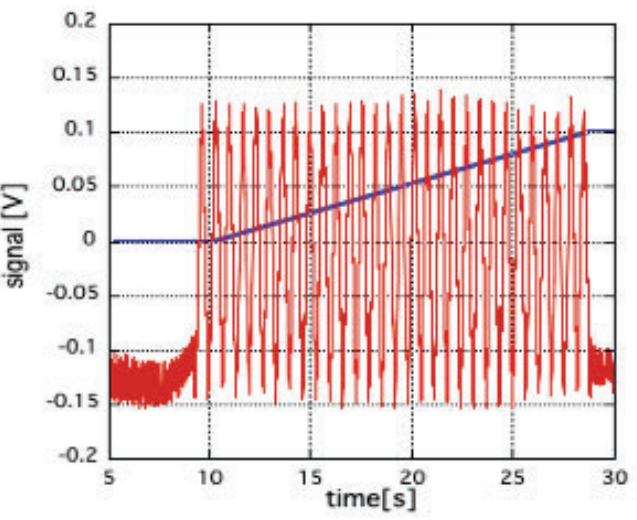

(b)

Figure 3. (a) Schematic diagram of the length measurement test, (b) temporal variation of the demodulated phase(red trace) and frequency of the beat signal (blue trace)

For long distance measurement, single-mode optical fibers are used as the optical path. 1.55- $\mu$ m single-frequency light source from a fiber-delivered micro external-cavity laser diode is divided into two by a 10-dB optical coupler. A small portion of the light is frequency-shifted by using an in-line AOM, and is re-combined with the laser through another blanch. The recombined beam which contains two frequency components is transmitted through a delivering optical fiber, and the transmitted light is detected by a photodetector at the end of the fiber to make beat note. The detected beat note is mixed with the local oscillator to obtain demodulated phase of the transmitted beat. The AOM driving frequency and the local oscillator is synchronously swept, and the temporal variation of the demodulated phase is recorded (red trace in Fig.3(b)). By using an up- and down-counter, we can measure $N$ from the variation of the phase with the resolution of $N / 2$, and the optical length of the fiber is calculated from the equation (1). We measure a various kind of fiber length by using combinations of $1-\mathrm{km}, 4-\mathrm{km}$ and $25-\mathrm{km}$ fiber spools. The driving frequency is swept from $50 \mathrm{MHz}$ to $66 \mathrm{MHz}$, and the length of the fibers are measured with length resolution of $6.25 \mathrm{~m}$ because no miscounting is occurred during measurement. 
This length measurement method is applied to our angle measurement system as follows; after diffracted beam from the leader satellite hits the follower satellite, the AOD driving frequency is fixed to keep illuminating the follower satellite, and the frequency of the local oscillator is scanned by AOM1. The length resolution of this system can be improved by increasing the scanned frequency range $\Delta f$. In the case of our system, the maximum frequency scanning range is $100 \mathrm{MHz}$ which corresponds to the length resolution of $1.5 \mathrm{~m}$. The length resolution would be further improved by detecting residual phase in addition to $N$ counting.

\section{CONCLUSIONS}

We have developed 3-dimentional satellite pointing system, in which the 2-dementional angle and the distance between satellites can be measured. In the preliminary test, the angle precision of $0.003 \mathrm{mrad}$ and the length resolution of $6.25 \mathrm{~m}$ are achieved. Currently the breadboard model of this system has been developed which is installed on aluminum breadboard and plan to test at the long vacuum tube. This system has been developed for initial alignment of DECIGO project, and would be a key technology for large-size precision formation flying missions.

\section{REFERENCES}

[1] C.V.M Fridlung, F.Capacioni,'Infrared space interferometry-the DARWIN mission", Advances in Space Research 30, 2135-2145 (2002)

[2] C.Beichman, P.Lawson, O.Ley, A.Ahmed, S.Unwin, K.Johnston, "Status of the Terrestrial Planet Finder Interferometer (TPF-I)", Proc SPIE 6268 Advances in Stellar Interferometry, 62680S (2006)

[3] Grace Follow-on: B.Sheard, G.Heinzel, K.Danzmann, D.Shaddock, "Intersatellite laser ranging instrument for GRACE follow-on mission", Journal of Geodesy 86, 1083-1095 (2012)

[4] W.Cash, A.Shipley, S.Osterman, M.Joy, "Laboratory detection of X-ray fringes with grazing-incidence interferometer", Nature 407, 160 (2000)

[5] M.Bavdaz, D.Lumb, P.Gondoin et.al, "The XEUS X-ray telescope", Proc SPIE 6266 Space Telescopes and Instrumentation II: Ultraviolet to Gamma Ray 62661S (2006)

[6] H.Tsunemi, K.Torii, E.Miyata, M.Nomachi, H.Kunieda, Y.Ogasaki, M.Ito, "Formation flight all sky telescope (FFAST)", Proc. of SPIE $\mathbf{5 9 0 0}$ Optics for EUV, X-ray and g-ray astronomy II, 590000 (2005)

[7] S.G.Turyshev, B.Lane, M.Shao, A Girerd, "A serch for New Physics with BEACON Mission", arXiv:0805.4033vc1 (2008)

[8] S.G.Turyshev, M.Shao, K.Nordtvedt, “The laser astrometric test of relativity mission”, Class. Quantum Grav. 21, 2773-2799 (2004)

[9] B.P.Abbott et.al, “ Observation of Gravitational Waves from a Binary Black Hole Merger “, Phys. Rev. Lett 116, 061102, (2016)

[10] P.Amaru-Seoane et.al., "Low-frequency gravitational-wave science with eLISA/NGO", Class. Quantum. Grav. 29, 124016 (2012)

[11] N.Seto, S.Kawamura and T.Nakamura, "Possibility of direct measurement of the acceleration of the universe using laser interferometer gravitational wave antenna in space”, Phys.Rev.Lett., 87, 221103 , (2001)

[12] T.Nakamura et.al. "Pre-DECIGO can get the smoking gun to decide the astrophysical or cosmological origin of GW150914-like binary black holes," Prog. Theory Exp. Phys. 2016, 093 E01 (2016)

[13] M.Musha, Kei Akami, Aru Suemasa, "Nobel satellite positioning system by using acousto-optic diffretor", Proceeding of ICSO2018, (2018) 14C-4

[14] L.D.Smullin and G.Fiocco, "Optical echoes from the moon”, Nature 194, 1267 (1962)

[15] I.Coddington, W.C.Swan, L.Nenadovic, N.R.Newbury, "Rapid and precise absolute distance measurements at long range", Nature Photonics 3, 351-356 (209) 